\title{
Solvothermal Pathways Towards Transition Metal Oxides: Exploration and Optimisation
}

\author{
Alexej Michailovski* and Greta R. Patzke
}

\begin{abstract}
A systematic solvothermal access towards nanoscale molybdenum and tungsten oxides has been established. Firstly, a versatile precursor material is selected that offers an interesting spectrum of reaction products. In the next step, a wide variety of straightforward ionic additives are screened to reveal and optimise all solvothermal pathways emerging from the precursor material. Finally, the appropriate precursor-additive combinations can be selected for further studies, such as nanorod design or the fabrication of novel materials.
\end{abstract}

Keywords: Nanorods · Solvothermal synthesis · Transition metal oxides

\section{Research Goals}

The discovery of the carbon nanotubes in 1991 [1] initiated the rapid development of nanochemistry [2][3]. In this context, solvothermal methods [4-6] have attracted considerable research interest as well. They provide control of particle size and access to kinetically stable phases so that they are especially suitable for the production of nanomaterials [7-9]. The successful development of a future nanotechnology demands robust and flexible preparative procedures. A multitude of solvothermal syntheses has recently been developed to meet these needs. Unfortunately, the solvothermal process itself continues to remain a 'black box' system [4], because the standard autoclave techniques do not permit a direct monitoring of the reaction progress. It would be highly desirable to fully explore

\footnotetext{
*Correspondence: A. Michailovski

the preparative potential of solvothermal reactions by elucidating their mechanistic background, but the necessary in situ approaches often involve highly sophisticated and expensive equipment [10]. So we aim for the systematic and efficient exploration of solvothermal syntheses with standard laboratory methods. Our main activities are focused on nanoscale anisotropic transition metal oxides, especially molybdenum-, vanadium- and tungsten oxides. They offer a wide range of important technological applications, e.g. in catalysis or sensor technology [11-14]. The preparative flexibility of the solvothermal process facilitates manifold structural and morphological studies [15]. Fig. 1 presents some highlights from our previous work [16-20]: the entire product spectrum can be accessed via straightforward syntheses in gram-scale quantities.

\section{Systemising Solvothermal Reac- tions: Nanoscale Molybdenum and Tungsten Oxides}

Solvothermal syntheses offer a vast multitude of preparative techniques and reaction parameters. In order to introduce some straightforward synthetic guidelines, we have developed the following strategy:

- The first explorative stage is centred on the quest for a flexible transition metal oxide-based material that gives rise to a maximum number of products. The precursor materials should be air-stable and readily available.

- The preparative potential of the optimum precursor is then fully explored with a screening procedure encompassing a wide range of additive systems. Again, preparative effort and expenses should be kept low by choosing the most straightforward additives, such as acids or binary ionic compounds. The additives are classified with respect to the resulting products and their morphologies until a set of synthetic profiles and solvothermal pathways emerges.

- Finally, the most versatile additive systems are selected for further morphological optimisation processes and follow-up studies including elementary mechanistic investigations (e.g. quenching/dilution techniques or reaction monitoring in glass ampoules). The systematic approach towards molybdenum oxide nanorods illustrates this threestep procedure (Fig. 2) [16][17].

\section{Example 1: Steering the Course of a Solvothermal Reaction Towards Molybdenum Oxide Nanorods \\ Step 1: Selecting the Precursor Ma-} terial

The right choice of the precursor material is crucial for the synthesis of molybdenum oxide nanorods, and yellow molybdic acid, $\mathrm{MoO}_{3} \cdot 2 \mathrm{H}_{2} \mathrm{O}$, has been proven a quite versatile educt. Whereas other starting materials, e.g. the closely related dehydration product $\mathrm{MoO}_{3} \cdot 0.33 \mathrm{H}_{2} \mathrm{O}$ or $\mathrm{MoO}_{3}$, can be employed for the growth of mm-sized molybdate crystals, yellow molybdic acid provides an especially convenient access to the desired molybdenum oxide nanofibres. 


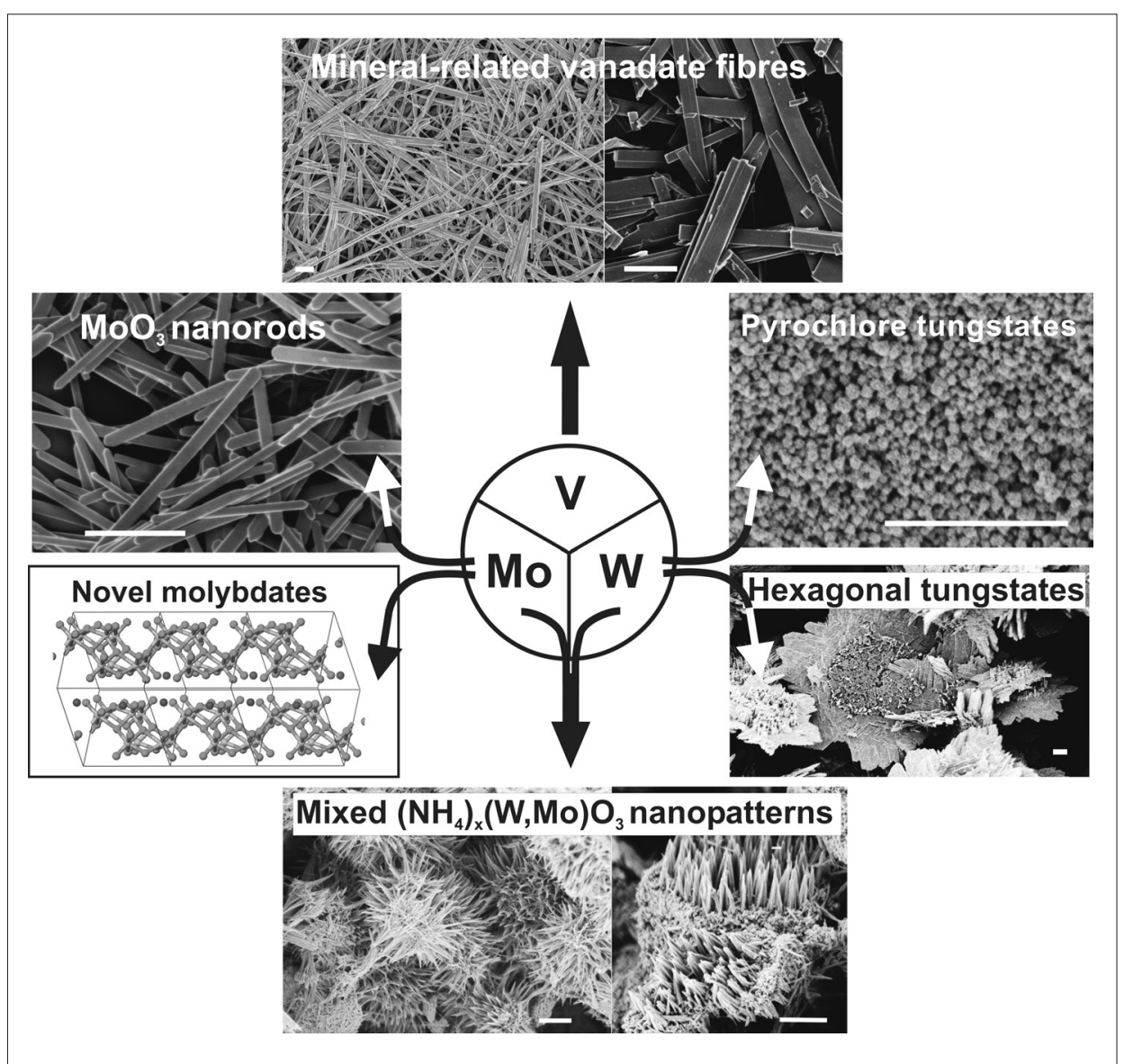
tems

\section{Step 2: Screening the Additive Sys-}

Readily available acids and the entire alkali/alkaline earth halide series were selected for a screening procedure with yellow molybdic acid. Applying the optimum reaction conditions revealed during step 1 , experiments were conducted for 2 days at $180{ }^{\circ} \mathrm{C}$ in a standard Teflon-lined steel autoclave $\left(1 \mathrm{mmol} \mathrm{MoO}_{3} \cdot 2 \mathrm{H}_{2} \mathrm{O}\right.$ and $2 \mathrm{ml}$ of $1 \mathrm{M}$ halide or $4.4 \mathrm{M}$ acid solution, respectively). As a result, a wide spectrum of molybdates emerged [17] and the most characteristic additive profiles are summed up briefly:

- $\mathbf{M o O}_{3}$ nanorods [8][9]: Rod-shaped products are exclusively formed in the presence of either neutral/acidic solutions or with the chlorides/bromides of the lighter alkali/alkaline earth cations (Li, Na, Mg, Ca).

- Hexagonal molybdates: The chlorides/bromides of the heavier alkali cations (K-Cs) and $\mathrm{Ba}$ favour the formation of microscale hexagonal molybdates with a channel structure and inhibit the formation of $\mathrm{MoO}_{3}$ nanorods. The hexagonal barium molybdenum bronze represents the first example of an alkaline earth-based hexagonal molybdate [17].

- Alkali tetramolybdates and fluoromolybdates: When alkali fluorides/iodides are applied as additives, the an- ionic part of the additive now drastically influences the course of the reaction, and mm-sized crystals of novel tetramolybdate and fluoromolybdate modifications emerge.

\section{tems}

Step 3: Exploring the Additive Sys-

Apart from the initial target of nanorod formation, the three main synthetic pathways now give rise to a diversity of additional research perspectives such as the structural characterisation of the novel alkali fluoromolybdates $\mathrm{Rb}_{6} \mathrm{Mo}_{8} \mathrm{O}_{26} \mathrm{~F}_{2} \cdot 6 \mathrm{H}_{2} \mathrm{O}$ and $\mathrm{Cs}_{6} \mathrm{Mo}_{8} \mathrm{O}_{26} \mathrm{~F}_{2}$. The formation of $\mathrm{MoO}_{3}$ nanorods was studied with respect to their formation pathways and morphology control. The individual dehydration steps occurring in acidic and neutral solvothermal media were revealed, and the according intermediates were subjected to EXAFS spectroscopy [16]. These investigations are currently expanded into a real in situEXAFS monitoring of the rod growth process. On the other hand, chemical factors triggering the formation of $\mathrm{MoO}_{3}$ nanorods can quickly be investigated with $\mathrm{NaCl}$ or $\mathrm{NaBr}$ as additive substances, because the sodium cation represents a borderline case, switching between the formation of rods and hexagonal sodium molybdates. The rod morphology can finally be affected by adjusting the reaction time/temperature and the additive combination/con-
Fig. 1. Selected product groups emerging from the systematic solvothermal fabrication of molybdenum-, vanadium- and tungsten oxides (scale bar $=1 \mu \mathrm{m}$ ).

centration. Fig. 2 illustrates how alkali and alkaline earth chlorides influence the aspect ratio of the rods, and these effects can be iteratively fine-tuned by further parameter variations. Depending on the solvothermal treatment chosen, the average $\mathrm{MoO}_{3}$ rod diameters can be varied between 80 and 500 $\mathrm{nm}$, whereas their lengths range from 600 $\mathrm{nm}$ to $44 \mu \mathrm{m}$.

A second example - taken from the rich chemistry of tungsten oxides - demonstrates detailed options for nanoscale morphology control (Fig. 3).

\section{Example 2: Morphology Tuning of Hexagonal Tungstate Nanorods}

Step 1: Choosing the Right Tungsten-based Precursor is a Pivotal Decision

Ammonium metatungstate exhibits a remarkable preparative flexibility that outperforms other potential educts such as tungstic acid. This precursor works best when subjected to the following solvothermal routine: an acidic solution of ammonium metatungstate is treated in a standard autoclave at $180{ }^{\circ} \mathrm{C}$ for $2-6$ d. Further parameter adjustments generate a series of products, encompassing $\mathrm{WO}_{3}$ nanoplatelets, hierarchically grown ammonium pyrochlore tungstate nanospheres and nanoscale hexagonal tungstates [18][19]. The latter were selected for detailed morphology studies. 
Step 2: The Screening Results Obtained with $\mathrm{MoO}_{3} \cdot 2 \mathrm{H}_{2} \mathrm{O}$ (cf. Example 1) Hold the Key to Selecting the Appropriate Class of lonic Additives

Alkali halides emerged as productive additives, and particularly the chloride anion adopts the role of a 'spectator anion' that does not interfere with morphology studies. Consequently, the alkali chlorides were combined with ammonium metatungstate and the effect of the individual cations on the nanoscale morphology of hexagonal tungstates was investigated. The solvothermal treatment was conducted for $2 \mathrm{~d}$ at $180{ }^{\circ} \mathrm{C}$ in a slightly acidified $1 \mathrm{M}$ solution of the alkali chloride. All cations are incorporated into the nanostructured products. They replace the ammonium cations in the W/O-hexagonal framework structure of $\left(\mathrm{NH}_{4}\right)_{0.26} \mathrm{WO}_{3}$ which is formed in an additive-free environment. In the next step, the additives were classified with respect to their capability of nanorod formation. Their synthetic profiles are quite distinct:

- LiCl: The standard precursor concentration affords hexagonal tungstate nanorods. Diluted precursor solutions favour the formation of flower-like shapes with nanorods growing perpendicular to the surface in a dendritic fashion.

- KCl: This additive does not permit straightforward morphology tuning so that considerable optimisation efforts were required to transform ammonium metatungstate into hexagonal potassium tungstate rods with microscale lengths and diameters below $100 \mathrm{~nm}$.

- RbCl and CsCl: The nanoscale hexagonal tungstates grown in the presence of the heavier alkali cations exhibit a characteristic cylindrical appearance (ca. $500 \mathrm{~nm}$ in length and $300 \mathrm{~nm}$ in diameter). These cylinders are further subdivided into stacked nanorods with diameters well below $30 \mathrm{~nm}$.

- NaCl: This additive is most suitable for the growth of hexagonal tungstate rods and has thus been singled out for further studies.

Step 3: A Few Extra Optimisation Steps with $\mathrm{NaCl}$ Lead to the Target Nanorods

Dilution approaches turned out to be the best method for adjusting the shape of the hexagonal tungstate rods (Fig. 3). The smallest rods produced with the standard routine exhibit diameters around $30 \mathrm{~nm}$ and lengths in the $100 \mathrm{~nm}$ range. The latter can be increased far into the microscale by choosing the appropriate precursor concentration, whilst the rod diameters are kept in the 100-300 $\mathrm{nm}$ range. Other parameters (reaction temperature or additive concentration) did not exert a comparable directing effect on the rod morphology.

\section{Conclusions}

The synthetic approach outlined in the examples can be extended to other systems, and it was applied for the growth of fibrous vanadates as well (Fig. 1, top). Again, the distinct synthetic profiles of the alkalibased additives were employed: whereas sodium-based additives, for example, favour the formation of synthetic mineral fibres, their lithium-based analogues yield mineral-related rods with considerably altered structures. Furthermore, the controlled fabrication of mixed nanoscale oxides can be derived from previous knowledge: the two most versatile precursor materials, $\mathrm{MoO}_{3} \cdot 2 \mathrm{H}_{2} \mathrm{O}$ (Example 1) and ammonium metatungstate (Example 2), were subjected to a common solvothermal reaction, and mixed $\left(\mathrm{NH}_{4}\right)_{\mathrm{x}}(\mathrm{W}, \mathrm{Mo}) \mathrm{O}_{3}$ nanorods emerged as a result [20]. These newly formed rods exhibit characteristic hierarchical nanoscale growth patterns, and the appropriate precursor combination is mandatory for their formation.

\section{Perspectives}

We systemised solvothermal reactions by exploring a wide range of precursor-additive interactions, followed by exploiting the synthetic potential of selected optimum combinations. The initial screening work is done without the help of combinatorial

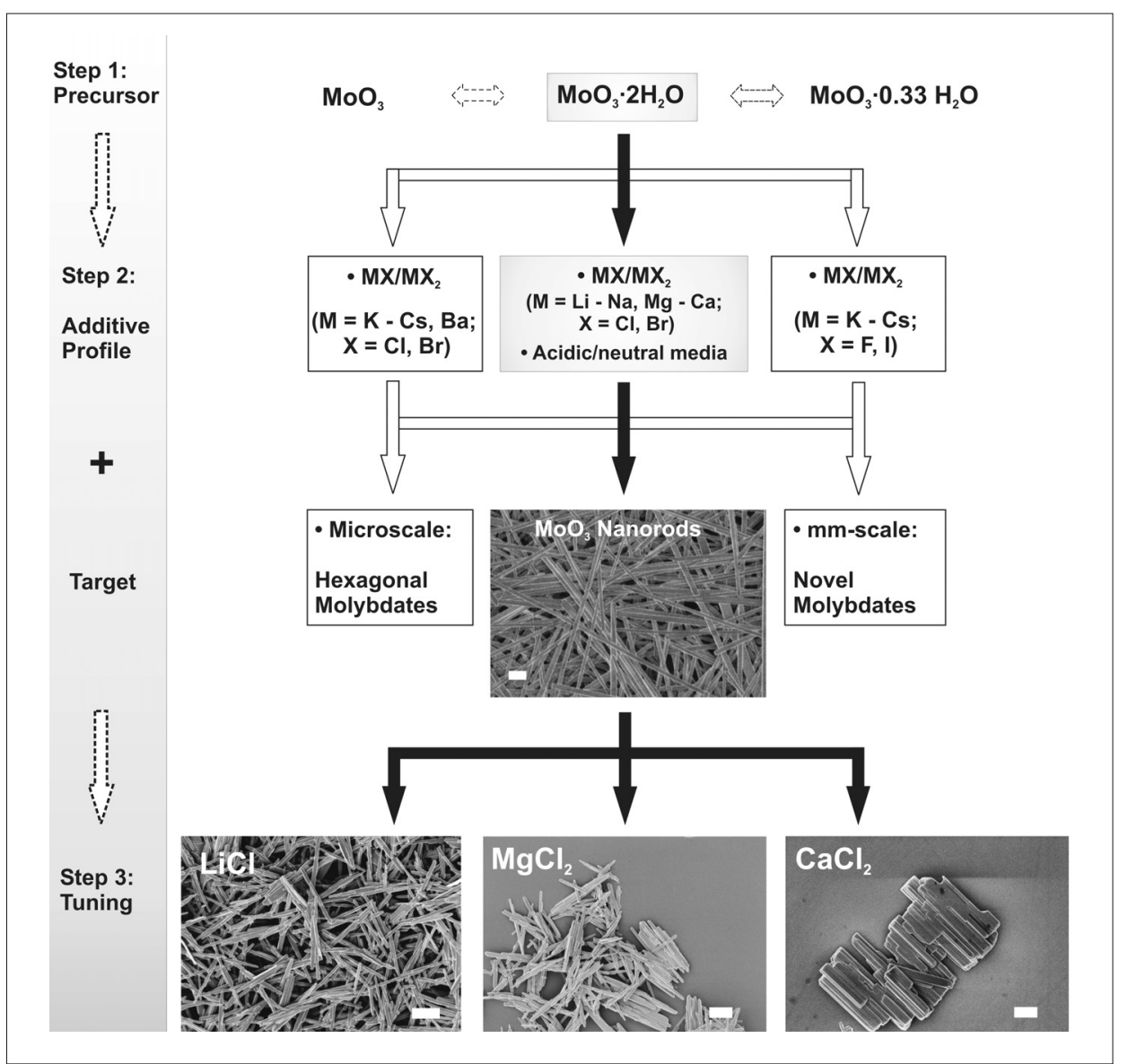

Fig. 2. Example 1: A systematic solvothermal approach towards fibrous $\mathrm{MoO}_{3}$ (scale bar $=1 \mu \mathrm{m})$. 


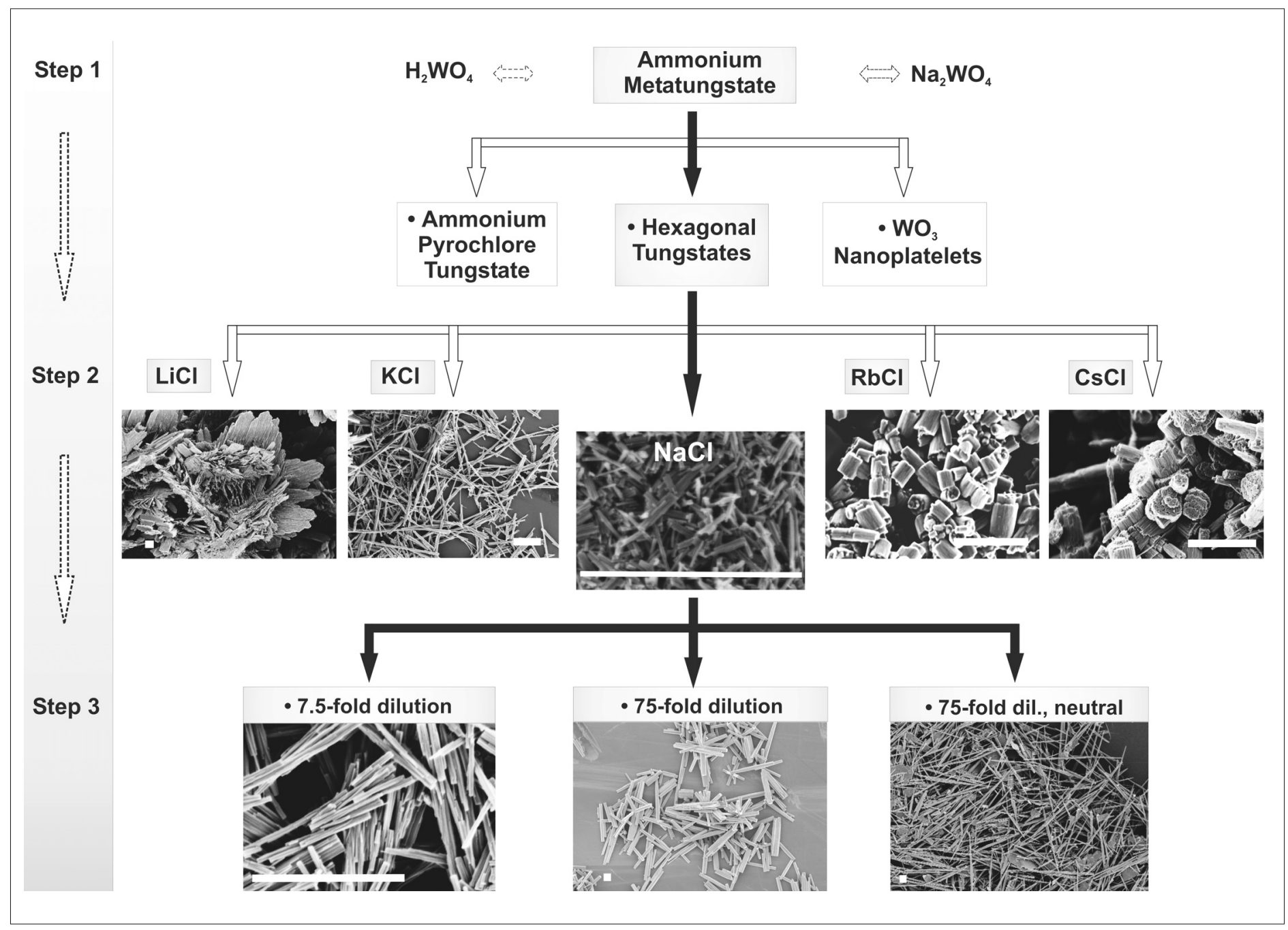

Fig. 3. Example 2: Morphology studies on hexagonal tungsten oxides (scale bar $=1 \mu \mathrm{m}$ ).

methods so that considerable preparative effort is required. The benefit of working under real conditions in gram quantities, however, outweighs this drawback: there are no extra scale-up problems to be faced and a single experiment usually provides enough material for various characterisation methods. The investigations are gradually extended over the whole series of transition metal oxides. In the end, a tailored solvothermal 'toolbox' system could result that would provide morphology control of nanoparticles. Our work might be regarded as a contribution to the challenging task of introducing design approaches into solid state chemistry.

\section{Acknowledgements}

The authors thank Prof. Dr. R. Nesper (Laboratory of Inorganic Chemistry, ETH Zürich) for his steady interest and the generous support of this work. Our research was funded by the ETH Zürich, by the Swiss National Science Foundation (MaNEP - Materials with Novel Electronic Properties), and by the National Research Program 'Supramolecular Functional Materials'.
[1] S. Iijima, Nature 1991, 354, 56.

[2] C.N.R. Rao, A.K. Cheetham, J. Mater. Chem. 2001, 11, 2887.

[3] R. Tenne, Prog. Inorg. Chem. 2001, 50, 269.

[4] R.I. Walton, Chem. Soc. Rev. 2002, 31, 230.

[5] K. Byrappa, M. Yoshimura, Handbook of Hydrothermal Technology, Noyes, Park Ridge, N. J., 2001.

[6] G.R. Patzke, F. Krumeich, R. Nesper, Angew. Chem. Int. Ed. 2002, 41, 2446.

[7] F. Krumeich, H.-J. Muhr, M. Niederberger, F. Bieri, B. Schnyder, R. Nesper, J. Am. Chem. Soc. 1999, 121, 8324.

[8] M. Niederberger, F. Krumeich, H.-J. Muhr, M. Müller, R. Nesper, J. Mater. Chem. 2001, 11, 1941.

[9] X.W. Lou, H.C. Zeng, Chem. Mater. 2002 $14,4781$.

[10] R.I. Walton, F. Millange, R.I. Smith, T.C. Hansen, D. O'Hare, J. Am. Chem. Soc. 2001, 123, 12547.

[11] A. Baiker, P. Dollenmeier, A. Reller, $J$. Catal. 1987, 103, 394.

[12] T. Chirayil, P.Y. Zavalij, M.S. Whittingham, Chem. Mater. 1998, 10, 2629.
[13] M. Niederberger, M.H. Bartl, G.D. Stucky, J. Am. Chem. Soc. 2002, 124, 13642.

[14] P.G. Dickens, A.C. Halliwell, D.J. Murphy, M.S. Whittingham, Trans. Faraday Soc. 1971, 67, 794.

[15] S. Mann, Angew. Chem. Int. Ed. 2000, 39, 3392.

[16] G.R. Patzke, A. Michailovski, F. Krumeich, R. Nesper, J.-D. Grunwaldt, A. Baiker, Chem. Mater. 2004, 16, 1126.

[17] A. Michailovski, F. Krumeich, G.R. Patzke, Helv. Chim. Acta, in print.

[18] A. Michailovski, F. Krumeich, G.R. Patzke, J. Solid State Chem., submitted.

[19] A. Michailovski, F. Krumeich, G.R. Patzke, Mater. Res. Bull., submitted.

[20] A. Michailovski, F. Krumeich, G.R. Patzke, Chem. Mater., in print. 